Research Article

\title{
SAR Target Recognition Using Improved Sparse Representation with Local Reconstruction
}

\author{
Li Ma (iD \\ School of Information Engineering, China University of Geosciences, Beijing, Beijing 100083, China \\ Correspondence should be addressed to Li Ma; 1004185206@cugb.edu.cn
}

Received 16 July 2021; Revised 31 July 2021; Accepted 5 August 2021; Published 11 August 2021

Academic Editor: Bai Yuan Ding

Copyright (c) $2021 \mathrm{Li} \mathrm{Ma}$. This is an open access article distributed under the Creative Commons Attribution License, which permits unrestricted use, distribution, and reproduction in any medium, provided the original work is properly cited.

\begin{abstract}
In order to handle the problem of synthetic aperture radar (SAR) target recognition, an improved sparse representation-based classification (SRC) is proposed. According to the sparse coefficient vector resulting from the global dictionary, the largest coefficient in each class is taken as the reference. Then, the surrounding neighborhoods of the sample with the largest coefficient are selected to construct the optimal local dictionary in each training class. Afterwards, the samples in the local dictionary are used to reconstruct the test sample to be identified. Finally, the decision is made according to the comparison of the reconstruction errors from different classes. In the experiments, the proposed method is verified based on the moving and stationary target acquisition and recognition (MSTAR) dataset. The results show that the proposed method has performance advantages over existing methods, which demonstrates its effectiveness and robustness.
\end{abstract}

\section{Introduction}

Synthetic aperture radar (SAR) is capable of measuring highresolution images for effective ground observation and surveillance. Image interpretation technologies represented by SAR target recognition are widely used in military and civilian fields. SAR target recognition is a typical image pattern recognition problem, which aims to extract and classify the target of interest in SAR images $[1,2]$. In order to improve the comprehensive performance of SAR target recognition, researchers extensively use advanced image feature extraction and classification algorithms for experimentation and verification. There are many types of features applied to SAR target recognition, including geometric shape features, projection transformation features, and electromagnetic scattering features. The geometric shape features describe the two-dimensional shape distribution of the target, such as area and contour [3-10]. The projection transformation features use mathematical projection or signal transformation algorithms to extract stable characteristics of the original images [11-16]. Electromagnetic scattering features describe the backscattering characteristics of the target in a specific radar frequency band, typically including the scattering centers [17-20] and polarizations [21]. In the classification stage, a suitable classifier is selected to confirm the class of the extracted features. Early classification strategies were mainly based on the idea of the nearest neighbors, such as K-nearest neighbor (KNN) classifier [11]. With the development of pattern recognition technologies, new classifiers such as support vector machine (SVM) [22, 23], multilayer perceptron (MLP) [12], and adaptive boosting (AdaBoost) [24] emerged. In recent years, the deep learning technology has become a new favorite in the field of image interpretation and has also been widely used in SAR target recognition [25-30]. Sparse representation-based classification (SRC) derived from compressive sensing theory was also widely used in pattern recognition and image classification [31-37]. Researchers introduced SRC into SAR target recognition and verified its feasibility. Since then, more works have continued to improve the overall recognition performance by optimizing the solution algorithm and decision-making mechanism [32-36].

Compared with other classifiers, SRC uses a linear fitting idea to evaluate the similarity between the test sample and each training class, so no pretraining is required. In addition, it is not difficult to find from the results of the existing 
literature that the sparse representation itself has a certain degree of robustness to the common extended operating conditions (EOCs) $[38,39]$ in SAR target recognition such as noise corruption and partial occlusion. Therefore, SRC has broad application prospects in SAR target recognition. In this paper, the traditional SRC is improved to enhance the performance in SAR target recognition. First, in the global dictionary, the test sample is reconstructed by SRC, and the sparse coefficient vector is obtained. Afterwards, the optimal local samples are selected in each classes according to a certain criterion. The criterion takes the sample with the largest coefficient as the reference and selects its surrounding neighborhoods to construct a local dictionary. Finally, the test sample is optimally reconstructed on the local dictionaries from different classes to obtain their corresponding reconstruction errors. The target class of the test sample is finally decided based on the principle of the minimum error. In the experiments, based on the moving and stationary target acquisition and recognition (MSTAR) dataset, the proposed method is tested under the standard operating condition (SOC) and three EOCs (configuration variance, depression angle difference, and noise corruption). Experimental results show that the proposed method can achieve superior performance over some existing methods in all four typical scenarios, verifying its effectiveness and robustness.

\section{SRC}

The sparse representation is based on the theory of compressive sensing and analyzes the characteristics of the sample by linearly representing the unknown sample on an overcomplete dictionary. Wright et al. first applied SRC in face recognition [31], that is, to determine the category of the test sample based on the reconstruction error of each class calculated based on the sparse representation coefficients. Specifically, a global dictionary $A=\left[A_{1}, A_{2}, \ldots, A_{C}\right] \in R^{d \times N}$ composed of multiple training classes is first constructed, where $A_{i}$ represents the $N_{i}$ atoms corresponding to the training samples in the $i$ th class. For the test sample $y$ to be identified, equation (1) is employed to perform the sparse linear represented:

$$
\begin{aligned}
& \widehat{x}=\underset{x}{\arg \min }\|x\|_{0}, \\
& \text { s.t. }\|y-A x\|_{2}^{2} \leq \varepsilon,
\end{aligned}
$$

where $x$ is the sparse coefficient vector to be solved and $\varepsilon$ is the set error threshold.

Since the direct solution of the optimization problem in equation (1) is very complicated, researchers tried to obtain high-confidence approximate solutions through the principle of equivalent approximation. For example, in [31], the $\ell_{1}$ norm was used to replace the original $\ell_{0}$ norm to convert the problem into a convex optimization one, which is easier to solve. In [32], an orthogonal matching pursuit algorithm (OMP) was developed based on a greedy mechanism to improve the overall solution efficiency. According to the solved sparse coefficient vector, the target class of the test sample can be judged according to its distributions in different classes. In [33], the decision was made according to the energy of nonzero coefficients in different classes. Among many principles, the criterion based on the minimum reconstruction error is the most widely used. The basic idea is to linearly reconstruct the test samples with samples of each class and then calculate the reconstruction error as follows:

$$
r(i)=\left\|y-A_{i} x_{i}\right\|_{2}^{2}, \quad(i=1,2, \ldots, C),
$$

where $x_{i}$ is the coefficient vector distributed on the $i$ th class and $r(i)$ is the reconstruction error of the $i$ th class to the test sample. Finally, the target class of the test sample can be determined by comparing the errors from different classes.

Although the decision criteria in the traditional SRC have certain validity, their characterization ability for each class is not sufficiently exploited. In the minimum reconstruction error criterion, all samples in each class are used for reconstruction. In fact, due to the azimuthal sensitivity of SAR images, the training samples related to the test sample should share a similar azimuth angle. Therefore, in order to obtain a better reconstruction result, the test sample should be reconstructed and analyzed on the local dictionary.

\section{Improvement of SRC for Target Recognition}

3.1. Improved SRC with Local Reconstruction. This paper makes some improvement on traditional SRC for SAR target recognition. Before implementing the traditional SRC, the atoms of each class in the dictionary are arranged in ascending azimuth order to ensure that the azimuths between adjacent samples are the closest. Afterwards, the global sparse coefficients are solved according to equation (1). Then, the optimal local dictionary is selected and constructed in each class. Taking the $i$ th class as an example, the atom with the maximum coefficient is used as the reference sample, and the surrounding neighborhood samples are chosen. Because SAR images are sensitive to azimuth, it is generally believed that they can maintain a high correlation within the interval of $\pm 5^{\circ}$. Therefore, this paper refers to this criterion in selecting surrounding samples. When the azimuth error is lower than $5^{\circ}$ with the reference sample, the candidate is incorporated into the local dictionary. Based on the local dictionary, this paper performs the optimal representation of the test sample class by class as follows:

$$
\widehat{\alpha}=\arg \min \left\{\left\|y-A_{L} \alpha_{L}\right\|_{2}^{2}+\lambda\left\|\alpha_{L}\right\|_{2}^{2}\right\},
$$

where $A_{L}$ represents the local dictionary selected on a certain class, $\alpha_{L}$ is the corresponding coefficient vector, and $\lambda$ is the regularization coefficient. The above optimization has the following analytical solution:

$$
\widehat{\alpha}=\left(A_{L}^{T} A_{L}+\lambda * I\right)^{-1} A_{L}^{T} y,
$$

where $I$ represents the unit matrix.

Compared with the traditional SRC mechanism, the optimization problem in equation (3) emphasizes the reconstruction accuracy. In fact, in the local dictionary, the sparsity constraint is not established, and the optimal 
reconstruction is more effective. Finally, according to the coefficient vector solved on each class, the corresponding reconstruction error for the test sample can be solved according to equation (2). Finally, the target class of the test sample is determined according to the principle of the minimum error.

3.2. Target Recognition Procedure. According to the above algorithms, the basic process of the recognition method proposed in this paper is shown in Figure 1. It can be further decomposed into the following steps:

Step 1: the test sample is processed by SRC based on the global dictionary formed by all the training samples and the sparse coefficient vector is solved

Step 2: the optimal local dictionary for each class is established according to Section 3.1

Step 3: the optimal reconstruction of the test sample is performed on the local dictionary of each class and the corresponding reconstruction error is calculated

Step 4: the target class of the test sample is determined according to the principle of the minimum error

In the specific implementation process, the principal component analysis (PCA) is used to extract feature vectors for all training and test samples so as to improve the overall classification efficiency. The OMP algorithm is used to solve the sparse coefficient vector resulting from the global dictionary.

\section{Experiments}

4.1. MSTAR Dataset. The performance of the proposed method is tested based on the MSTAR dataset. The dataset has been the authoritative benchmark for the testing and verification of SAR target recognition methods since its public release in the 1990s. Figure 2 shows the target in the dataset, including tanks, armored vehicles, and transport vehicles. The SAR image of each type of target contains omnidirectional azimuths and several depression angles, so that various conditions can be flexibly set to carry out experiments. According to existing literature, Table 1 gives a typical experimental setup, using SAR images of all the 10 types of targets at $17^{\circ}$ and $15^{\circ}$ depression angles as training and testing sets, respectively. In particular, the test samples of BMP2 and T72 targets have more configurations than their training samples (different configurations are marked by the notations in the parentheses). In general, under the experimental condition in Table 1, the differences between the training and test sets are relatively small, which can be approximated as SOC. In addition, according to the diversity of the image samples of the MSTAR dataset, several EOCs can be set or simulated, such as configuration variance, depression angle variance, and noise interference.

During the experiment, the proposed method is compared with several types of existing methods, focusing on comparison with traditional SRC-based methods, including the SRC1 method in [32], the SRC2 method in [33], and the SRC3 method in [34]. These three types of methods either adopt different decision-making mechanisms or introduce new solution constraints. In addition, a new method based on CNN is also set up in the comparison method, i.e., ESENet, proposed in [29]. The follow-up experiments specifically include 4 types: 1 SOC and 3 EOCs. SOC focuses on evaluating the basic recognition performance of the method, and EOCs verify the robustness of the method, mainly the reliability in complex scenarios.

4.2. SOC. At first, the performance of the proposed method is tested under SOC using the training and test sets in Table 1. In this case, the similarities between the test sample and the training sample are relatively high, so the difficulty of the recognition problem is relatively low. However, since Table 1 involves 10 types of targets, the correct classification still faces certain challenges. Figure 3 shows the recognition results of the proposed method under SOC. In the confusion matrix, the diagonal element is the correct recognition rate of the corresponding target. Considering the correct recognition samples of 10 types of targets, the average recognition rate of the proposed method in this paper reaches $99.04 \%$, which shows its effectiveness. Table 2 compares the recognition results of various methods under this condition. The recognition rate of the proposed method is significantly higher than those of the traditional SRC-based methods, indicating that proper local dictionary selection and optimal reconstruction can improve the performance of SAR target recognition. Compared with the ESENet method, the recognition rate of the proposed method is slightly higher. Due to the configuration differences that occurred in BMP2 and T72 in the test set, the adaptability of the trained networks has declined to a certain extent. In summary, the proposed method has certain performance advantages under SOC.

4.3. EOC-1: Configuration Variance. Table 3 sets the training and test samples under the condition of configuration variance, including 3 types of targets. Among them, the test samples and samples of BMP2 and T72 targets are from different configurations. Table 4 shows the average recognition rates of different methods under this situation. The comparison shows that the proposed method can maintain the highest performance under configuration variance, showing its superior robustness. Compared with the other three types of SRC-based methods, this paper optimizes the local dictionary in the global sparse coefficients and performs the optimal reconstruction class by class, which further improves the effectiveness of the proposed method. Under configuration variance, there are only small differences in the local structure of the targets between the test and training samples. The reconstruction in a single class rather than the global dictionary is helpful to discover such subtle differences, thereby improving the recognition accuracy. Compared with the results under SOC, the recognition performance of the ESENet method decreases the most significantly, mainly because the influence of the configuration variances is further aggravated at this time. 


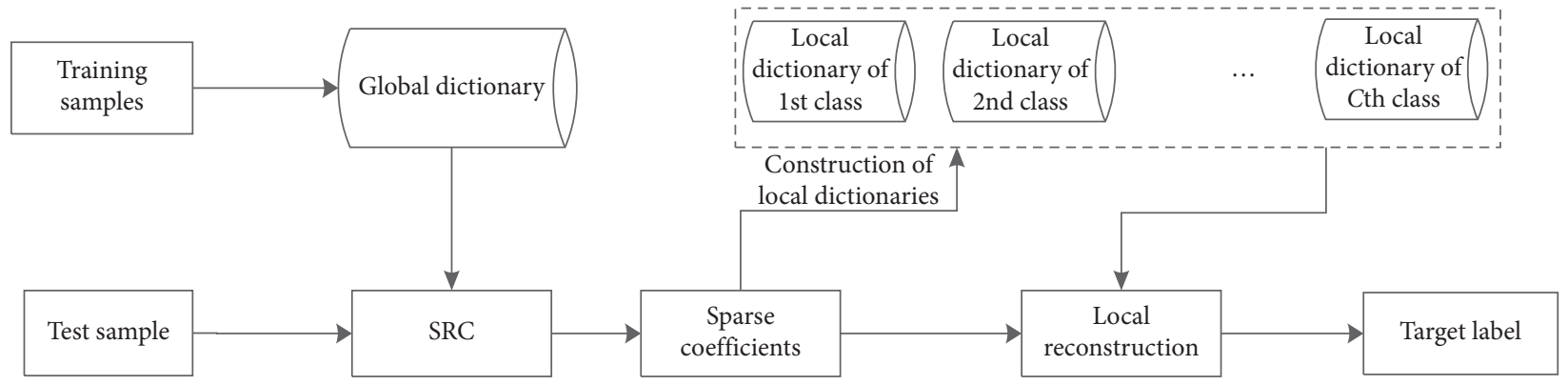

FIGURE 1: Procedure of SAR target recognition based on improved SRC.

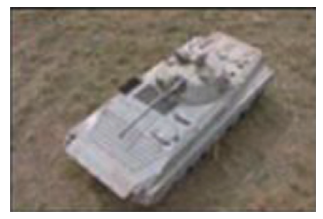

(a)

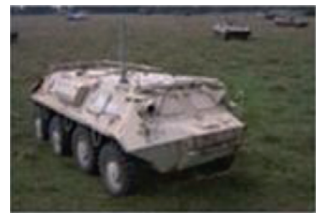

(f)

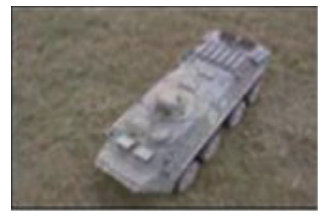

(b)

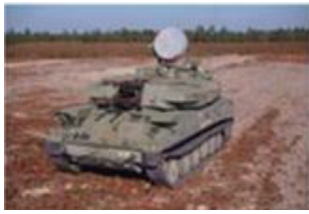

(g)

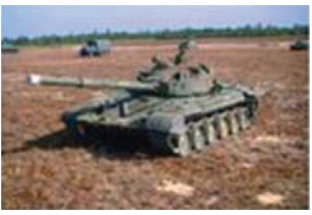

(c)

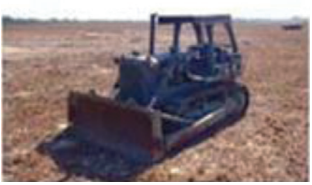

(h)

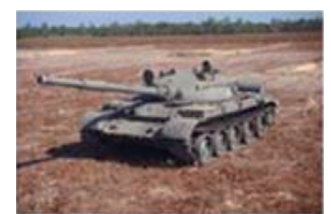

(d)

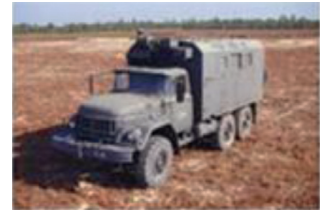

(i)

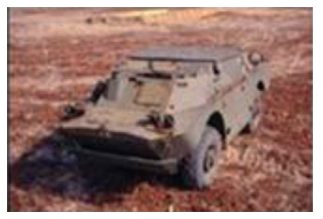

(e)

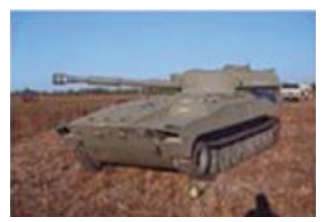

(j)

Figure 2: Optical images of targets in MSTAR dataset [35]. (a) BMP2, (b) BTR70, (c) T72, (d) T62, (e) BRDM2, (f) BTR60, (g) ZSU23/4, (h) D7, (i) ZIL131, and (j) 2 S1.

TABle 1: Training and test samples under SOC.

\begin{tabular}{|c|c|c|c|c|c|c|c|c|c|c|}
\hline Target class & BMP2 & BTR70 & T72 & T62 & BRDM2 & BTR60 & ZSU23/4 & D7 & ZIL131 & $2 S 1$ \\
\hline Training set & $233(9563)$ & 233 & $232(132)$ & 299 & 298 & 256 & 299 & 299 & 299 & 299 \\
\hline Test set & $587(9563,9566, \mathrm{C} 21)$ & 233 & $582(132,812, \mathrm{~S} 7)$ & 273 & 274 & 195 & 274 & 274 & 274 & 274 \\
\hline
\end{tabular}

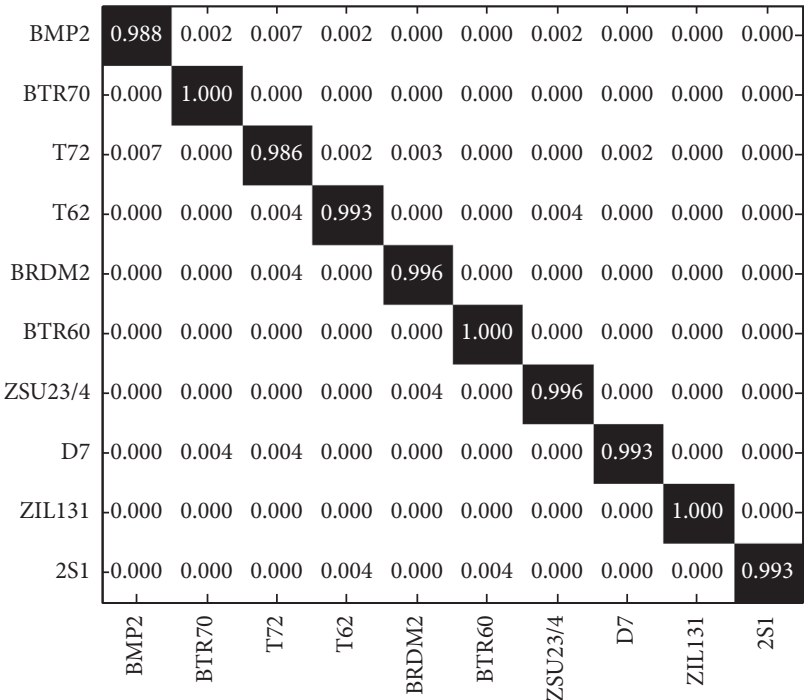

Figure 3: Confusion matrix of the proposed method under SOC.
TABLE 2: Comparison of performance under SOC.

\begin{tabular}{lc}
\hline Method & Average recognition rate (\%) \\
\hline Proposed & 99.04 \\
SRC1 & 96.12 \\
SRC2 & 96.63 \\
SRC3 & 97.21 \\
ESENet & 98.78 \\
\hline
\end{tabular}

4.4. EOC-2: Depression Angle Variance. The MSTAR dataset also includes SAR images of several types of targets at multiple different depression angles. As shown in Table 5, the training samples are images of 3 types of targets, i.e., 2S1, BRDM2, and ZSU23/4, from the depression angle of $17^{\circ}$; the test samples are from the depression angles of $30^{\circ}$ and $45^{\circ}$, respectively. The large depression angle variance leads to a decrease in the similarity between the test and training samples, which brings about certain obstacles to correct recognition. Table 6 compares the average recognition rates 
TABLE 3: Experimental condition under different configurations.

\begin{tabular}{lcccc}
\hline \multirow{2}{*}{ Target class } & \multicolumn{2}{c}{ Training set $\left(17^{\circ}\right)$} & \multicolumn{2}{c}{ Test set $\left(15^{\circ}\right)$} \\
\hline BMP2 & Configuration & Sample amount & Configuration & Sample amount \\
T72 & 9563 & 233 & $9566 \mathrm{c} 21$ & 196 \\
BTR60 & & & & 196 \\
T62 & 132 & 232 & $812 \mathrm{~s} 7$ & 195 \\
& 7532 & 256 & 7532 & 191 \\
\end{tabular}

TABle 4: Comparison of performance under configuration variances.

\begin{tabular}{lc}
\hline Method & Average recognition rate $(\%)$ \\
\hline Proposed & 98.52 \\
SRC1 & 94.76 \\
SRC2 & 96.08 \\
SRC3 & 97.34 \\
ESENet & 97.72 \\
\hline
\end{tabular}

TABLE 5: Training and test samples under depression angle variance [37].

\begin{tabular}{lcccc}
\hline & Target class & 2 S1 & BRDM2 & ZSU23/4 \\
\hline \multirow{2}{*}{ Training set } & Depression angle & $17^{\circ}$ & $17^{\circ}$ & $17^{\circ}$ \\
& Sample amount & 299 & 298 & 299 \\
\hline \multirow{4}{*}{ Test set } & Depression angle & $30^{\circ}$ & $30^{\circ}$ & $30^{\circ}$ \\
& Sample amount & 288 & 287 & 288 \\
& Depression angle & $45^{\circ}$ & $45^{\circ}$ & $45^{\circ}$ \\
& Sample amount & 303 & 303 & 303 \\
\hline
\end{tabular}

TABle 6: Comparison of performance at different depression angles.

\begin{tabular}{lcc}
\hline Method & \multicolumn{2}{c}{ Average recognition rate $(\%)$} \\
& $30^{\circ}$ & $45^{\circ}$ \\
\hline Proposed & 95.45 & 73.28 \\
SRC1 & 93.52 & 66.37 \\
SRC2 & 94.03 & 67.78 \\
SRC3 & 94.48 & 69.12 \\
ESENet & 95.03 & 67.26 \\
\hline
\end{tabular}

of various methods at two test depression angles. The average recognition rate of the proposed method is $95.45 \%$ and $73.28 \%$ at the elevation angles of $30^{\circ}$ and $45^{\circ}$, respectively, which has advantages compared with other methods. Especially at $45^{\circ}$ with a notable depression difference, the advantages of the proposed method are more significant. Through proper local dictionary selection and optimal reconstruction, the characterization ability of different classes for the current test sample can be investigated to the greatest extent, so it can better adapt to the situation of depression angle variances.

4.5. EOC-3: Noise Corruption. In order to quantitatively test the noise robustness of the proposed method under different signal-to-noise ratios (SNR), the test samples in

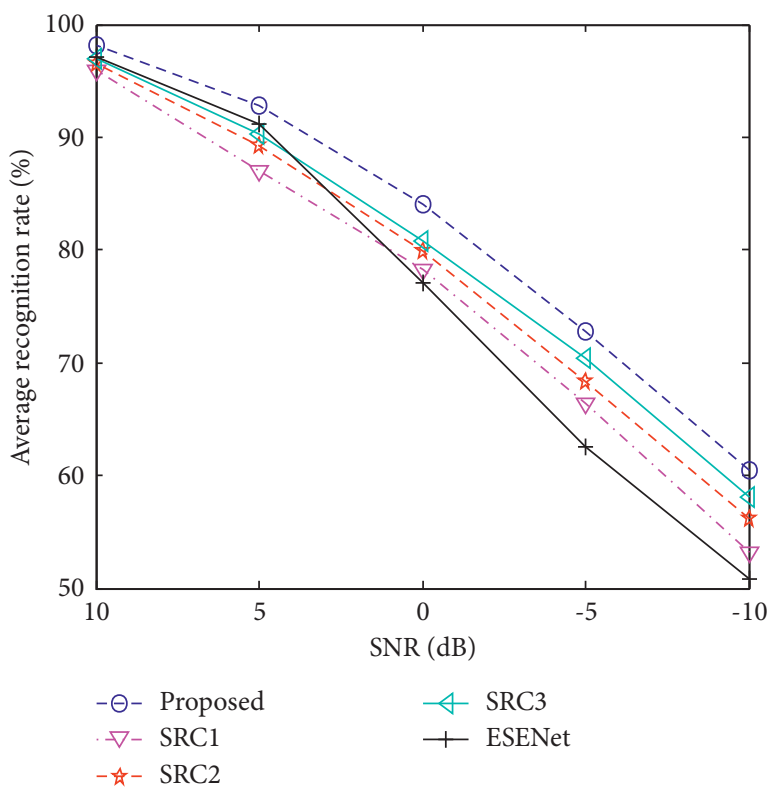

FIGURE 4: Comparison of performance under noise corruption.

Table 1 are used as the benchmark and different degrees of Gaussian white noise are added to them according to the ideas in [19]. Figure 4 plots the recognition rate curves of various methods with the change of the SNR. The proposed method can achieve the highest average recognition rate at each noise level, showing its better noise robustness. Compared with the ESENet method, several types of methods based on SRC are generally more robust, especially in the case of low SNR, verifying that sparse representation has a certain robustness to noise interference. The proposed method examines the representation ability of each class on a reliable local dictionary and can deal with noise corruption more effectively through the optimal reconstruction process.

\section{Conclusion}

The paper proposes an improved SRC for the SAR target recognition. On the basis of the global sparse coefficients obtained from traditional SRC, the local dictionary is constructed according to each training class. Considering the azimuthal sensitivity of SAR images, this paper takes the sample with the largest correlation coefficient in each class as the reference and selects some of its neighborhood samples with approaching azimuths to construct the local dictionary 
with the strongest representation ability. Based on the local dictionary, the linear fitting is performed on the test sample according to the idea of optimal reconstruction, and finally the target class of the test sample is determined by comparing the reconstruction errors resulting from different classes. Based on the MSTAR dataset, the proposed method is tested under 4 typical conditions. The experimental results reflect that the proposed method can maintain superior performance under both SOC and EOCs, which proves its effectiveness and robustness for SAR target recognition.

\section{Data Availability}

The dataset used in this paper is publicly available.

\section{Conflicts of Interest}

The author declares that there are no conflicts of interest regarding the publication of this paper.

\section{References}

[1] K. El-Darymli, E. W. Gill, and P. McGuire, "Automatic target recognition in synthetic aperture radar imagery: a state-ofthe-art review," IEEE Access, vol. 4, pp. 6014-6058, 2016.

[2] B. Ding and G. Wen, "Target reconstruction based on 3-D scattering center model for robust SAR ATR," IEEE Transactions on Geoscience and Remote Sensing, vol. 56, no. 7, pp. 3772-3785, 2018.

[3] B. Ding and G. Wen, "A region matching approach based on 3-D scattering center model with application to SAR target recognition," IEEE Sensors Journal, vol. 18, no. 11, pp. 4623-4632, 2018.

[4] M. Amoon and G. A. Rezai-rad, "Automatic target recognition of synthetic aperture radar (SAR) images based on optimal selection of Zernike moments features," IET Computer Vision, vol. 8, no. 2, pp. 77-85, 2014.

[5] S. Gishkori and B. Mulgrew, "Pseudo-Zernike moments based sparse representations for SAR image classification," IEEE Transactions on Aerospace and Electronic Systems, vol. 55, no. 2, pp. 1037-1044, 2019.

[6] X. Zhang, Z. Liu, and S. Liu, "Sparse coding of 2D-slice Zernike moments for SAR ATR," International Journal of Remote Sensing, vol. 38, no. 2, pp. 412-431, 2017.

[7] P. Bolourchi, H. Demirel, and S. Uysal, "Target recognition in SAR images using radial Chebyshev moments," Signal Image \& Video Processing, vol. 11, no. 6, pp. 1-8, 2017.

[8] C. Shan, B. Huang, and M. Li, "Binary morphological filtering of dominant scattering area residues for SAR target recognition," Computational Intelligence and Neuroscience, vol. 2018, Article ID 9680465, 3 pages, 2018.

[9] G. C. Anagnostopoulos, "SVM-based target recognition from synthetic aperture radar images using target region outline descriptors," Nonlinear Analysis, vol. 71, no. 2, pp. e2934-e2939, 2009.

[10] J. Tan, X. Fan, S. Wang et al., "Target recognition of SAR images by partially matching of target outlines," Journal of Electromagnetic Waves and Applications, vol. 33, no. 7, pp. 865-881, 2019.

[11] A. K. Mishra, "Validation of PCA and LDA for SAR ATR," in Proceedings of the IEEE TENCON, pp. 1-6, Hyderabad, India, November 2008.
[12] A. K. Mishra and T. Motaung, "Application of linear and nonlinear PCA to SAR ATR," in Proceedings of the IEEE 25th International Conference Radioelektronika (RADIOELEKTRONIKA), pp. 349-354, Pardubice, Czech Republic, April 2015.

[13] Z. Cui, Z. Cao, and J. Yang, "Target recognition in synthetic aperture radar images via non-negative matrix factorisation," IET Radar, Sonar \& Navigation, vol. 9, no. 9, pp. 1376-1385, 2015.

[14] G. Dong and G. Kuang, "Classification on the monogenic scale space: application to target recognition in SAR image," IEEE Transactions on Image Processing, vol. 24, no. 8, pp. 2527-2539, 2015.

[15] G. Dong, G. Kuang, and N. Wang, "SAR target recognition via joint sparse representation of monogenic signal," IEEE Journal of Selected Topics in Applied Earth Observations and Remote Sensing, vol. 8, no. 7, pp. 3316-3328, 2015.

[16] X. Miao and Y. Liu, "Target recognition of SAR images based on complex bidimensional empirical mode decomposition," Scientific Programming, vol. 10, Article ID 6642316, 2021.

[17] H. C. China, R. L. Moses, and L. C. Potter, "Model-based classification of radar images," IEEE Transactions on Information Theory, vol. 46, no. 5, pp. 1842-1854, 2000.

[18] B. Ding, G. Wen, and J. Zhong, "Robust method for the matching of attributed scattering centers with application to synthetic aperture radar automatic target recognition," Journal of Applied Remote Sensing, vol. 10, no. 1, Article ID 16010, 2016.

[19] B. Ding, G. Wen, J. Zhong, and C. X. Yang, "A robust similarity measure for attributed scattering center sets with application to SAR ATR," Neurocomputing, vol. 219, pp. 130-143, 2017.

[20] B. Ding, G. Wen, and X. Huang, "Target recognition in synthetic aperture radar images via matching of attributed scattering centers," IEEE Journal of Selected Topics in Applied Earth Observations and Remote Sensing, vol. 10, no. 7, pp. 3334-3347, 2017.

[21] W. Yang, J. Lu, and Z. Cao, "A new algorithm of target classification based on maximum and minimum polarizations," in Proceedings of the CIE International Conference on Radar, pp. 1-4, Beijing, China, October 2001.

[22] Q. Zhao and J. C. Principe, "Support vector machines for SAR automatic target recognition," IEEE Transactions on Aerospace and Electronic Systems, vol. 37, no. 2, pp. 643-654, 2001.

[23] H. Liu and S. Li, "Decision fusion of sparse representation and support vector machine for SAR image target recognition," Neurocomputing, vol. 113, pp. 97-104, 2013.

[24] Y. Sun, Z. Liu, and S. Todorovic, "Adaptive boosting for SAR automatic target recognition," IEEE Transactions on Aerospace and Electronic Systems, vol. 43, no. 1, pp. 112-125, 2007.

[25] M. Kang, K. Ji, X. Leng, X. Xing, and H. Zou, "Synthetic aperture radar target recognition with feature fusion based on a stacked autoencoder," Sensors, vol. 17, no. 1, p. 192, 2017.

[26] S. Chen, H. Wang, F. Xu, and Y. Q. Jin, "Target classification using the deep convolutional networks for SAR images," IEEE Transactions on Geoscience and Remote Sensing, vol. 54, no. 6, pp. 1685-1697, 2016.

[27] J. Zhao, Z. Zhang, and W. Yu, "A cascade coupled convolutional neural network guided visual attention method for ship detection from SAR images," IEEE Access, vol. 6, pp. 50693-50708, 2018.

[28] R. Min, H. Lan, and Z. Cao, "A gradually distilled CNN for SAR target recognition,” IEEE Access, vol. 7, pp. 42190-42200, 2019. 
[29] L. Wang, X. Bai, and F. Zhou, "SAR ATR of ground vehicles based on ESENet," Remote Sensing, vol. 11, no. 11, p. 1316, 2019.

[30] P. Zhao, K. Liu, and H. Zou, "Multi-stream convolutional neural network for SAR automatic target recognition," Remote Sensing, vol. 10, no. 9, p. 1473, 2018.

[31] J. Wright, A. Y. Yang, and A. Ganesh, "Robust face recognition via sparse representation," IEEE Transactions on Pattern Analysis and Machine Intelligence, vol. 31, no. 2, pp. 210-227, 2009 .

[32] J. J. Thiagaraianm, K. N. Ramamurthy, and P. Knee, "Sparse representations for automatic target classification in SAR images," in Proceedings of the 4th International. Symposium. Communication., Control Signal Processing, pp. 1-4, Limassol, Cyprus, March 2010.

[33] L. Zhang, Z. Tao, and B. Wang, "SAR image target recognition using kernel sparse representation based on reconstruction coefficient energy maximization rule," in Proceedings of the IEEE ICASSP, pp. 2369-2373, Shanghai, China, March 2016.

[34] X. Xing, K. Ji, H. Zou, and J. Sun, "Sparse representation based SAR vehicle recognition along with aspect angle," Science World Journal, vol. 2014, Article ID 834140, 10 pages, 2014.

[35] L. Yu, L. Wang, and Y. Xu, "Combination of joint representation and adaptive weighting for multiple features with application to SAR target recognition," Scientific Programming, vol. 2021, Article ID 9063419, 9 pages, 2021.

[36] C. Zhang, X. Jiang, and X. Liu, "Semi-supervised SAR ATR via multi-discriminator generative adversarial network," IEEE Sensors Journal, vol. 19, no. 17, pp. 7525-7533, 2019.

[37] B. Chen, X. Ma, and C. Liu, "SAR target recognition based on joint sparse representation of complementary features," in Proceedings of the 2018 International Conference on Sensors, Signal and Image Processing, pp. 29-34, Athens, Greece, October 2018.

[38] J. R. Diemunsch and J. Wissinger, "Moving and stationary target acquisition and recognition (MSTAR) model-based automatic target recognition: search technology for a robust ATR," in Proceedings of the 5th SPIE Algorithms Synthetic Aperture Radar Image, vol. 3370, pp. 481-492, Orlando, FL, USA, September 1998

[39] E. Keydel, S. Lee, and J. Moore, "MSTAR extended operating conditions: a tutorial," in Proceedings of the SPIE, pp. 228-242, Cambridge, UK, December 1996. 